Kalpa Publications in Engineering
Volume 3, 2020, Pages 223-230
Proceedings of International Sym-
posium on Applied Science 2019

\title{
Meshless Radial Point Interpolation Method For Hyperelastic Materials
}

\author{
Trong Khiem Bui ${ }^{1}$, Tuong Lam Nguyen $\mathrm{Vu}^{1}$, Thanh Nha Nguyen ${ }^{1}$, Tich \\ Thien Truong ${ }^{1}$ \\ ${ }^{1}$ Ho Chi Minh city University of Technology - VNUHCM \\ nhanguyen@hcmut.edu.vn
}

\begin{abstract}
Hyperelastic materials are special types of material that tends to behavior elastically when they are subjected to very large strains. These materials show not only the nonlinear material behavior but also the large deformation and stress-strain relationship is derived from a strain energy density function. Hyperelastic materials are widely used in many applications such as biological tissues, polymeric foams and moreover. Neo - Hookean is a material model for hyperelastic solid which contains only two material parameters: bulk modulus and shear modulus. In the field of numerical analysis, radial point interpolation method (RPIM) is a wellknown meshfree method based on Garlekin weak form. With the property of "free of mesh", the RPIM approach shows its advantage for large deformation problems. In this study, a meshless radial point interpolation method is applied to demonstrate elastic response of rubber-like materials based on the MooneyRivlin model. The obtained results are compared with the reference solutions given by other methods to verify the accuracy of the proposed method.
\end{abstract}

\section{Introduction}

Hyperelastic material is material which is used to represent large deformation behavior. Rubber, rubber - like materials and other polymer are hyperelastic materials. These materials are used widely in reality to approximate the material behavior of biological tissues, polymeric foams... Unlike metal, the stress - strain relation of this material is non - linear and really different between tension and compression. Because of this reason, the simulation of deformable behavior of hyperelastic material is much more complicate than the deformation of metal we know.

Throughout history, there are many numerical methods which were introduced to solve lots of mechanical problems such as finite element method, boundary element method, ... and they have gained great achievement. One of the newest methods and prove its advantage among others is the meshfree method. In meshfree method, the approximate solution for partial differential is obtained using a set of scattered nodes in the absence of mesh. Therefore, we construct shape functions for particular 
points using nodes in a small local domain, which is called the support domain. Mesh is no need in meshfree method, this means that no connectivity information between nodes is needed before the calculation, connectivity is defined during run as a part of computation. This is appropriate for large deformation problems which require remeshing in mesh based methods.

Today, some commercial software can solve the problem of hyperelasticity based on finite element method. Beside that, in research aspect, various meshfree methods have been developed and extended to deal with these problems. For example, "The meshless method for rubber hyperelastic material based on Yeoh mode type constitutive laws" [1] or "The Meshless Local Petrov-Galerkin Method for Large Deformation Analysis of Hyperelastic Materials" [2]. But the shape functions which were used in these methods not qualify for Kronecker - delta, thus having difficulty in handling boundary conditions.

In this paper, we select the radial point interpolation method [4], which has the Kronecker delta function and consistency property, to solve the problem of hyperelasticity. Until now, RPIM was applied to solve many mechanical problems such as "Radial point interpolation method for elastoplastic problems in Proceeding" [3], "A point interpolation meshless method based on radial basis functions" [4].

\section{RPIM Shape Function}

Consider $\mathrm{u}(\mathrm{x})$ be field variable function defined in the problem domain. The domain is represented by a set of arbitrarily distributed nodes in the problem domain and its boundary. Approximation for the function $\mathrm{u}(\mathrm{x})$ within a support domain at point $\mathrm{x}$ is given by:

$$
u^{h}(x)=\sum n i=1 R i(r) a i+\sum m j=1 p j(x) b j
$$

Where $R i(r)$ are the radial basis functions; $\mathrm{n}$ is the number of nodes in support domain; $\operatorname{Pj}(x)$ are the monomials; $\mathrm{m}$ is the number of polynomial basis functions; $b j$ are the corresponding coefficients for the polynomial basis functions $\operatorname{Pj}(x)$. Equation (1) can be written in the matrix form as:

$$
U_{s}=R_{0} a+P_{m} b
$$

Where the vector $U_{s}^{T}=\left[u_{1}, u_{2}, \ldots, u_{n}\right]$; the moment matrix of radial basis functions, $R_{0}$ is expressed as

$$
R_{0}=\left[\begin{array}{cccc}
R_{1}\left(r_{1}\right) & R_{2}\left(r_{1}\right) & \ldots & R_{n}\left(r_{1}\right) \\
R_{1}\left(r_{2}\right) & R_{2}\left(r_{2}\right) & \ldots & R_{n}\left(r_{2}\right) \\
\vdots & \vdots & \ddots & \vdots \\
R_{1}\left(r_{n}\right) & R_{2}\left(r_{n}\right) & \ldots & R_{n}\left(r_{n}\right)
\end{array}\right]
$$

the moment matrix of polynomial terms $P m$ is expressed as

$$
P_{m}^{T}=\left[\begin{array}{ccc}
1 & \ldots & 1 \\
x_{1} & \ldots & x_{n} \\
y_{1} & \ldots & y_{n} \\
\vdots & \vdots & \vdots \\
P_{m}\left(x_{1}\right) & \ldots & P_{m}\left(x_{n}\right)
\end{array}\right]
$$

In order to have an unique solution, the following constraints are imposed for the polynomial terms:

$$
\sum_{i=1}^{n} p_{j}\left(x_{i}\right) a_{i}={ }_{P_{m} T} a=0, \mathrm{j}=1,2, \ldots, \mathrm{m}
$$

Combining both equation (2) and (3), we obtain the following equation: 


$$
\left[\begin{array}{cc}
R_{0} & P_{m} \\
P_{m}^{T} & 0
\end{array}\right]\left[\begin{array}{l}
a \\
b
\end{array}\right]=\left[\begin{array}{c}
U_{s} \\
0
\end{array}\right]
$$

Or can be written as:

$$
\mathrm{G}\left[\begin{array}{l}
a \\
b
\end{array}\right]=\left[\begin{array}{c}
U_{s} \\
0
\end{array}\right]
$$

Substituting equation (5) into equation (1), we have:

$$
u(x)=R^{T}(x) a+p^{T}(x) b=\left[\begin{array}{ll}
R^{T}(x) & p^{T}(x)
\end{array}\right]\left[\begin{array}{l}
a \\
b
\end{array}\right]=\left[\begin{array}{ll}
R^{T}(x) & p^{T}(x)
\end{array}\right] G^{-1}\left[\begin{array}{c}
U_{s} \\
0
\end{array}\right]=\bar{\Phi}^{T}(x)\left[\begin{array}{c}
U_{s} \\
0
\end{array}\right]
$$

where we can express the RPIM shape functions as:

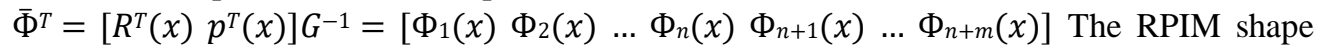

functions corresponding to nodal variables are:

$$
\Phi^{T}(x)=\left[\Phi_{1}(x) \Phi_{2}(x) \ldots \Phi_{n}(x)\right]
$$

Equation (1) can be written as:

$$
u(x)=\Phi^{T}(x) U_{s}=\sum_{i=1} \phi_{i} u_{i}
$$

\section{Deformation Of Hyperelastic Material}

\subsection{Deformation measures used in Hyperelastic material}

We suppose that a solid is subjected to a displacement field $u i(x k)$

- $\quad$ The deformation gradient and its Jacobian

$$
F_{i j}=\delta_{i j}+\frac{\partial x_{i}}{\partial x_{j}} \quad ; \quad J=\operatorname{det}(F)
$$

- The left Cauchy - Green deformation tensor

$$
B i j=F i k . F j k
$$

- $\quad$ Invariants of B

$$
\begin{gathered}
I_{1}=B_{k k} \\
I_{2}=\frac{1}{2}\left(I_{1}^{2}-B_{i k} B_{k i}\right) \\
\quad I_{3}=\operatorname{det}(B)=J^{2}
\end{gathered}
$$

- An alternative set of invariants of B 


$$
\begin{gathered}
\bar{I}_{1}=\frac{I_{1}}{J^{2 / 3}}=\frac{B_{k k}}{J^{2 / 3}} \\
\bar{I}_{2}=\frac{I_{2}}{J^{4 / 3}}=\frac{1}{2}\left(\bar{I}_{1}^{2}-\frac{B_{i k} B_{k i}}{J^{4 / 3}}\right) \\
J=\sqrt{\operatorname{det}(B)}
\end{gathered}
$$

\subsection{The stress-strain relations from the strain energy density}

The constitutive law for an isotropic hyperelastic material is defined by an equation relating the strain energy density of the material to the deformation gradient, or, for an isotropic solid, to the three invariants of the strain tensor

$$
\mathrm{W}(\mathrm{F})=\mathrm{U}\left(I_{1}, I_{2}, I_{3}\right)=\bar{U}\left(\bar{I}_{1}, \bar{I}_{2}, J\right)
$$

Stress - Strain relation in terms of $\bar{I}_{1}, \bar{I}_{2}, J$

$$
\sigma_{i j}=\frac{2}{J}\left[\frac{I_{1}}{J^{2 / 3}}\left(\frac{\partial \bar{U}}{\partial \bar{I}_{1}}+\bar{I}_{1} \frac{\partial \bar{U}}{\partial \bar{I}_{2}}\right) B_{i j}-\left(\bar{I}_{1} \frac{\partial \bar{U}}{\partial \bar{I}_{1}}+2 \bar{I}_{2} \frac{\partial \bar{U}}{\partial \bar{I}_{2}}\right) \frac{\delta_{i j}}{3}-\frac{1}{J^{4 / 3}} \frac{\partial \bar{U}}{\partial \bar{I}_{2}} B_{i k} B_{k j}\right]+\frac{\partial \bar{U}}{\partial J} \delta_{i j}
$$

For Generalized Neo - Hookean solid

$$
\bar{U}=\frac{\mu_{1}}{2}\left(\bar{I}_{1}-3\right)+\frac{\mu_{1}}{2}(J-1)^{2}
$$

where $\mu 1$ and $\mathrm{K} 1$ are material properties (for small deformations, $\mu 1$ and $\mathrm{K} 1$ are the shear modulus and bulk modulus of the solid, respectively). This is a rubber elasticity model, for rubbers with very limited compressibility, and should be used with $\mathrm{K} 1>>\mu 1$. The stress - strain relation follows as

$$
\sigma_{i j}=\frac{\mu_{1}}{J^{\frac{5}{3}}}\left(B_{i j}-\frac{1}{3} B_{k k} \delta_{i j}\right)+K_{1}(J-1) \delta_{i j}
$$

\section{Numerical Example}

In order to test the developed approach, two numerical examples are given for analyzing the large deformation of bodies (Cook's membrane and Curved beam). To generate the numerical results, the shear modulus $(\mu)$ is assumed to be $80.194 \mathrm{~N} / \mathrm{mm} 2$. Besides, the bulk modulus $(\kappa)$ is taken as 120.291 $\mathrm{N} / \mathrm{mm} 2$ in the compressible regime and $400889.806 \mathrm{~N} / \mathrm{mm} 2$ in nearly incompressible regime.

\subsection{Cook's membrane example}

This example is commonly selected to determine the effective of discretized formulations in bending problems. The membrane has a trapezoidal shape, the geometry and dimensions of are shown in Fig. 1. An edge traction load $f=32 \mathrm{~N} / \mathrm{mm} 2$ is given along the right edge and it is clamped along the left edge. 


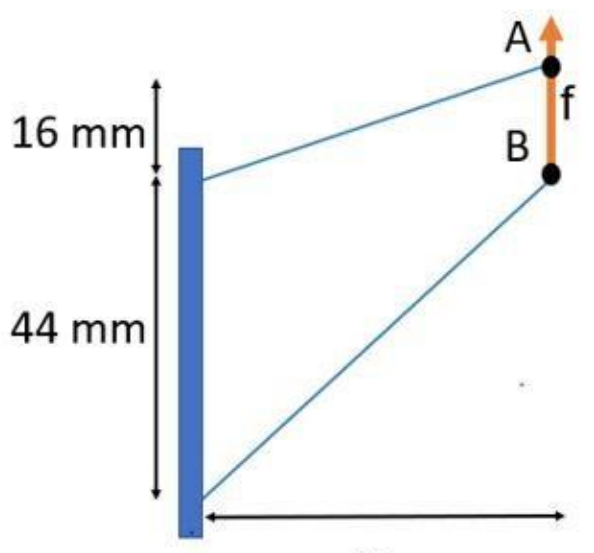

$48 \mathrm{~mm}$

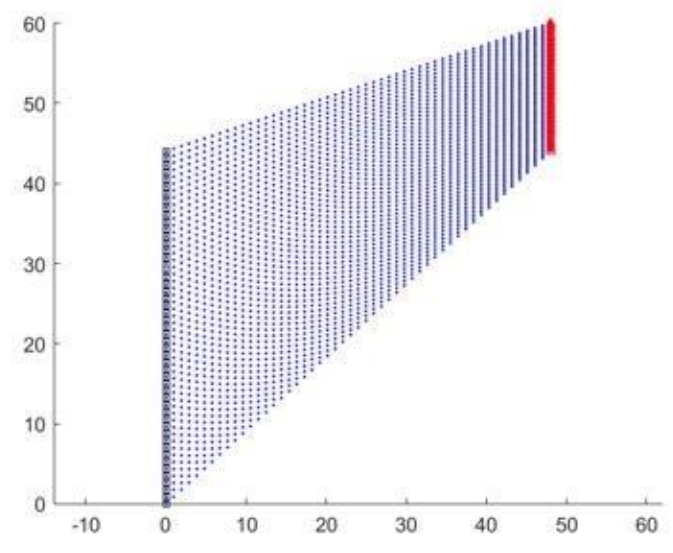

Figure 1: Schematic of the of Cook's Figure 2: RPIM mesh of Cook's membrane example membrane

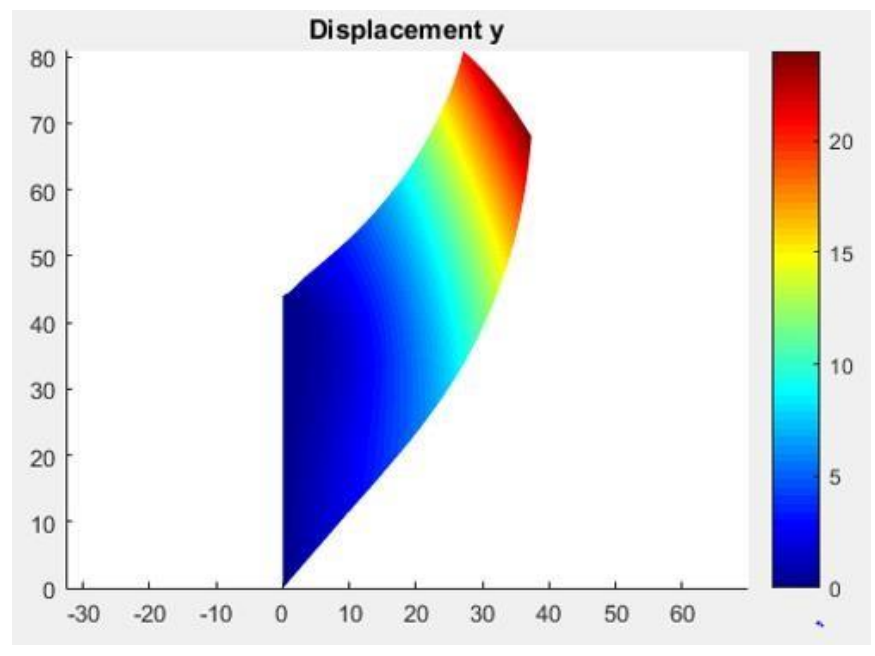

Figure 3: Deformed configuration of nearly - incompressible Cook's membrane

There are 2600 nodes used for the model (see Fig. 2). The plot in Fig. 3 shows the deformation of the nearly - incompressible membrane. It is observed that the maximum vertical displacement is obtained at point B. Table. 1 shows the comparison of the vertical displacements at point A and B between proposed RPIM and FEM results given by R. Hassani et al [5]. A good agreement between the present results and reference results is obtained. In addition, Fig. 4 is presented to show the convergence behavior of the method for the nearly-incompressible model.

To investigate the effect of the size of support domain on RPIM results for this nonlinear problem, several values of this parameter are chosen for computing. Table. 2 shows the results of displacement are more exactly when the support domain $\alpha$ is increased, however it takes more steps for calculating. 
Table 1. Comparison of the vertical displacement between RPIM and FEM

\begin{tabular}{|c|c|c|c|c|}
\hline & $\begin{array}{c}\text { FEM (ANSYS) } \\
(\mathrm{mm})\end{array}$ & RPIM (mm) & FEM [5] (mm) & ERROR (\%) \\
\hline$u_{y} A$ & 21.4 & 20.87 & 21.38 & 2.38 \\
\hline$u_{y} B$ & 24.94 & 24.01 & - & 3.84 \\
\hline
\end{tabular}

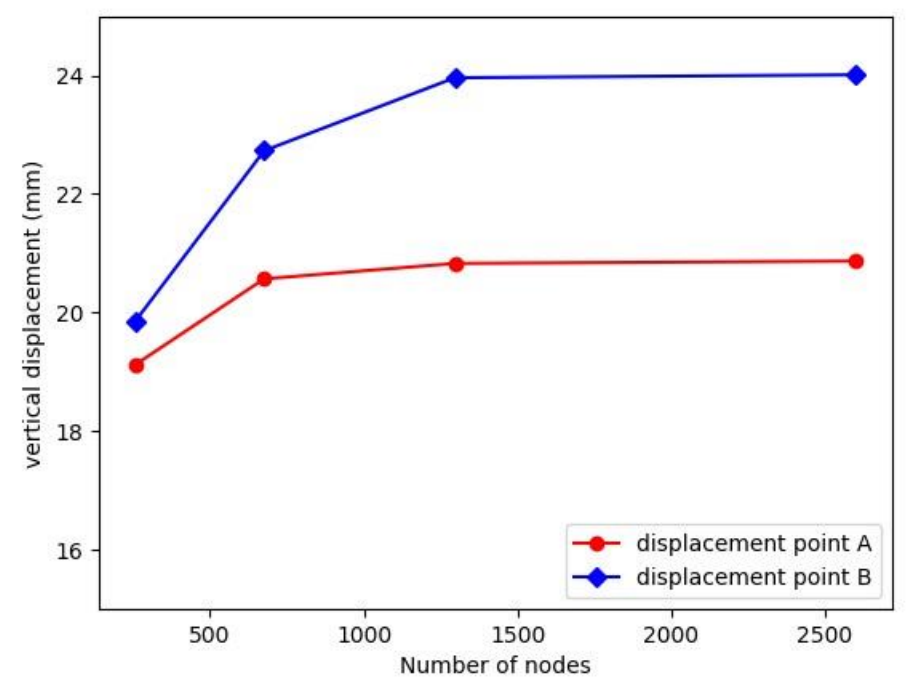

Figure 4: Vertical displacement of point $\boldsymbol{A}$ and B in nearly-incompressible Cook's membrane with the number of nodes for various values of distributed shearing force $f$

Table 2. Displacement with the size of support domain for various values

\begin{tabular}{|c|c|c|c|c|c|}
\hline & 1.7 & 1.8 & 1.9 & 2.0 & 2.1 \\
\hline$u_{y} A$ & 20.2003 & 20.3190 & 20.4656 & 20.4396 & 20.5492 \\
\hline$u_{y} B$ & 22.5630 & 22.7742 & 22.7409 & 23.1452 & 23.3611 \\
\hline
\end{tabular}

\subsection{Curved beam example}

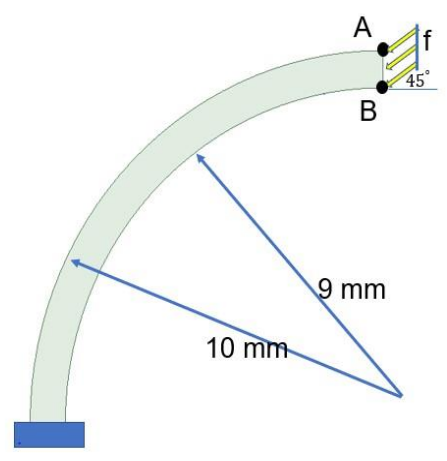

Figure 5: Schematic of the curved beam example 
In the second example, the behavior of a compressible curved beam subjected to a compression is investigated. According to Fig. 5, a quarter-circular curved beam is considered which is clamped along the lower edge. Plots in Fig. 6 display the total displacement distribution of the curved beam with various values of force $\mathrm{f}$.

To verify the nodal convergence, several nodal distributions are selected for computation. Charts in Fig. 7 show the convergence of the method for the compressible problem, it is reasonable to see that the higher values of force gives larger total displacement than others.

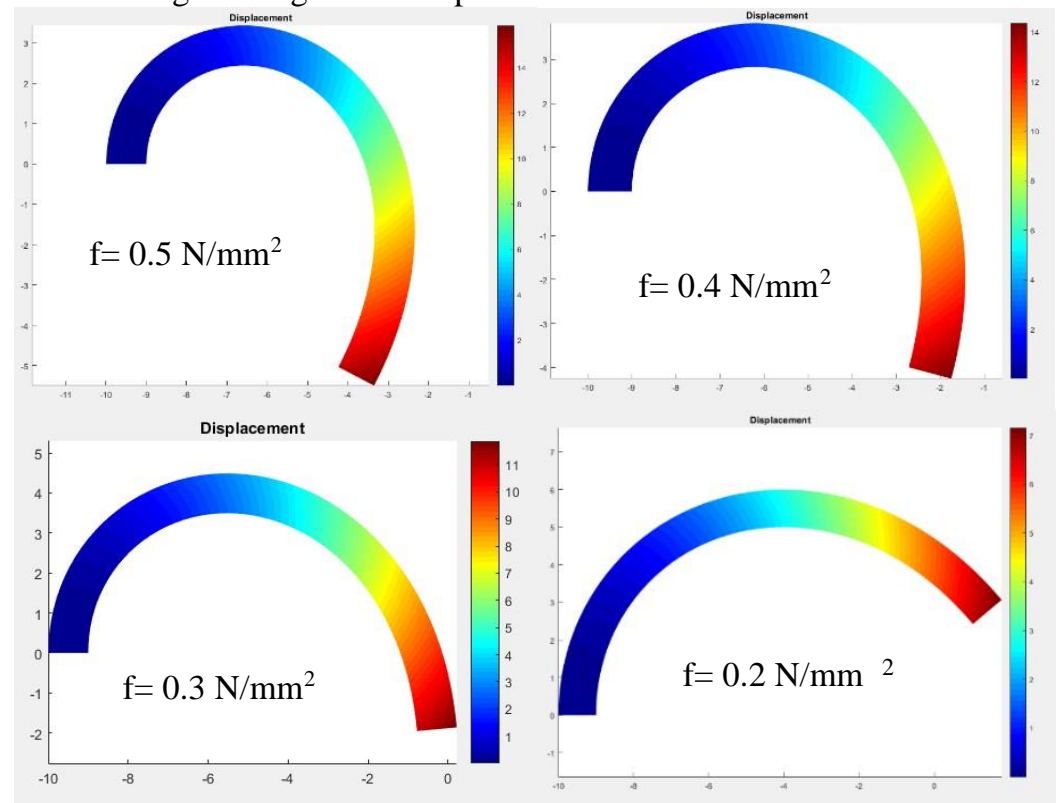

Figure 6: Deformed configuration of compressible curved beam with several values of distribution force $\mathrm{f}: 0.5$, $0.4,0.3,0.2(\mathrm{~N} / \mathrm{mm} 2)$

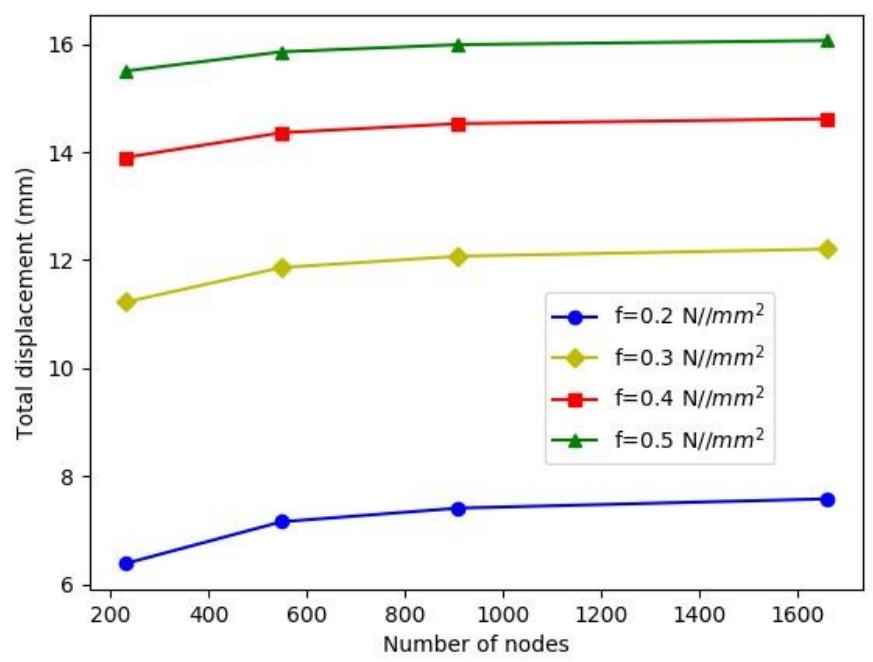

Figure 7: Total displacement of point A in compressible curved beam with the number of nodes for various values of distributed shearing force $f$ 


\section{Conclusions}

In this paper, the meshless radial point interpolation method is applied to solve the large deformation problem with hyperelastic material. These computational programs are developed in the Matlab programming language. The neo-Hookean model is used for the hyperelastic behavior of the material, several numerical examples in the nearly incompressible and compressible regimes were solved in order to show the validity and efficiency of the meshless approach. The proposed RPIM method can solve the problem with an acceptable accuracy. It was shown that the present method is effective in solving various nonlinear elasticity problems such as nearlyin compressible and compressible.

\section{Acknowledgments}

This research is support by Department of Engineering mechanics. The authors would like to thank all the advices from lecturers for their valuable comments and suggestions.

\section{References}

G.Zhao. "The meshless method for rubber hyperelastic material based on Yeoh mode type constitutive laws", Journal of Basic Science and Engineering, 17, (1), (2009), pp. 121-127

D. A. Hu. "The Meshless Local Petrov-Galerkin Method for Large Deformation Analysis of Hyperelastic Materials", International Scholarly Research Network, (2011)

J. G. Wang. "Radial point interpolation method for elastoplastic problems in Proceeding", 1st Structural Conference on Structural Stability and Dynamics, (2000)

J. G. Wang. "A point interpolation meshless method based on radial basis functions", International Journal for Numerical Methods in Engineering, (2002)

R. Hassani. "Large deformation analysis of $2 \mathrm{D}$ hyperelastic bodies based on the compressible nonlinear elasticity: A numerical variational method", International Journal of Non-Linear Mechanics, (2019) 